\title{
DEFORMATIONS OF LOG-LAGRANGIAN SUBMANIFOLDS OF POISSON MANIFOLDS
}

\begin{abstract}
ZIV RAN
ABSTRACT. We consider Lagrangian-like submanifolds in certain even-dimensional 'symplectic-like' Poisson manifolds. We show, under a suitable transversality hypothesis, that the pair consisting of the ambient Poisson manifold and the submanifold has unobstructed deformations, and that the deformations automatically preserve the Lagrangian-like property.
\end{abstract}

The study of holomorphic Lagrangian submanifolds of compact holomorphic symplectic manifolds and their deformation theory is well established (see e.g. [6] and references therein). Voisin [16] proved that pairs $(X, Y)$ consisting of a compact Kählerian symplectic manifold $X$ and a Lagrangian submanifold $Y$ have unobstructed deformations, i.e. an appropriate Kuranishi space is polydisc; and under these deformations $Y$ stays Lagrangian. See also [8].

Recently, refining some results of Goto [5] and Hitchin [7], we studied in [11] certain even-dimensional compact Kählerian Poisson manifolds called pseudosymplectic, from the point of view that they are analogous to symplectic manifolds. A Poisson structure $\Pi \in \mathrm{H}^{0}\left(X, \wedge^{2} T_{X}\right)$ on a complex manifold $X$ of dimension $2 n$ is said to be pseudo-symplectid] if it is almost everywhere nondegenerate. When that is the case, $\Pi$ will degenerate along an anticanonical divisor $D=\left[\Pi^{n}\right]$ called the Pfaffian of $\Pi$. We introduced a condition on $\Pi$ called P-normality, which says that $D$ has normal crossings and is smooth wherever $\Pi$ has corank exactly 2 . Roughly speaking, the P-normality condition means that $(X, \Pi)$ is locally a product of Poisson manifolds of the form (smooth surface, smooth anticanonical divisor). We showed under this condition that $(X, \Pi)$ has, in a strong sense, unobstructed deformations.

Date: September 13, 2018.

2010 Mathematics Subject Classification. 14J40, 32G07, 53D12, $53 \mathrm{D} 17$.

Key words and phrases. Poisson structure, symplectic structure, complex structure, Lagrangian submanifold, deformation theory, differential graded Lie algebra, Schouten algebra, log complex, mixed Hodge theory.

arxiv.org/1311.2656.

${ }^{1}$ As a referee suggests, 'log-symplectic' might be a better term; we also thank this referee for suggesting the term 'log-Lagrangian' used below. 
Here we consider an analogue of Lagrangian submanifolds in the Poisson setting. An $n$-dimensional closed submanifold $Y$ in a $2 n$-dimensional pseudo-symplectic manifold $(X, \Pi)$ is said to be log-Lagrangian if for all $y \in Y$, the conormal space $\breve{N}_{Y, y}$ is an isotroptic subspace for $\Pi$. Note that the log-Lagrangian condition implies that for all $y \in Y \backslash D$, i.e. all points $y \in Y$ - if any- where $\Pi$ is nondegenerate, $T_{Y, y}$ is a maximal isotropic subspace for the symplectic form $\Phi_{y}=\Pi_{y}^{-1}$.

As an immediate example (see also Example 6 below), if $S$ is a smooth surface endowed with an effective anticanonical divisor $C=[\Pi], \Pi \in H^{0}\left(O_{S}(-K)\right)$, there is a natural Poisson structure $\Pi^{[r]}$ on the Hilbert scheme $S^{[r]}$ (see [11]). If $B$ is any smooth curve on $S$, then $B^{(r)} \subset S^{[r]}$ is a log-Lagrangian submanifold.

In this paper we will restrict our attention to log-Lagrangians satisfying a transversality condition. Specifically, if $(X \Pi)$ is a pseudo-symplectic Poisson manifold with Pfaffian $D$, a $\log$-Lagrangian $Y$ is said to be transverse (to $D$ or to $\Pi$ if specification be needed) if $X$ is P-normal in a neighborhood of $Y$, and locally at every point $y \in Y \cap D$, the intersection $\bar{D}=D \cap Y$ is a normal-crossing divisor on $Y$ (this transversality condition holds vacuously if $D \cap Y$ is empty). When $Y$ is transverse, we have that $\check{N}_{Y, y}$ is isotropic for all $y \in Y$. In the Hilbert scheme example above, $B^{(r)}$ is transverse if $B$ is transverse to $C$; in fact, $D$ is smooth in a neighborhood of $D \cap B^{(r)}$.

Given $(X, \Pi)$ pseudo-symplectic Poisson and $Y \subset X$ log-Lagrangian, one may consider deformations of the triple $(X, \Pi, Y)$ with or without the condition that $Y$ stay log-Lagrangian. Let us call such deformations Poisson-Lagrange or Poisson respectively. One may also consider deformations of $Y$ in $X$, with $(X, \Pi)$ fixed, again with or without the Lagrangian condition; these will be named Lagrange or Hilbert respectively. In fact, we will prove that the deformation spaces with or without the Lagrangian condition are identical and smooth; in particular, the log-Lagrangian property is 'sticky', impossible to move away from. Thus, our main purpose is to prove

Theorem. Let $Y$ be a compact transverse log-Lagrangian submanifold of a Kählerian Poisson manifold $(X, \Pi)$ that is $P$-normal along $Y$. Then

(i) $Y$ has unobstructed Hilbert and Lagrange deformations in $X$, and these deformation spaces are identical;

(ii) if moreover $\Pi$ is P-normal everywhere and $X$ is compact, the triple $(X, \Pi, Y)$ has unobstructed Poisson-Lagrange and Poisson deformations, and these deformation spaces are identical.

This result includes the special case where $Y$ is empty, which is the main result of [11], as well as the special case where $\Pi$ is symplectic (so $D$ is empty: Voisin's theorem [16]). In fact, we will prove a more precise result (see Theorem 3 below). 
From a different viewpoint, some results on deformations of submanifolds of Poisson manifolds were obtained by Baranovsky, Ginzburg et al. [1].

\section{Précis of DEFORMATION THEORY}

1.1. Lie atom, SELA. A (dg) Lie atom [13] is the mapping cone associated to a Lie module homomorphism

$$
i: \mathfrak{g} \rightarrow \mathfrak{h}
$$

from a (differential graded) Lie algebra $\mathfrak{g}$, endowed with the left regular representation, to a g-module. A Lie atom is the special case corresponding to a 1-simplex of a semi-cosimplcial object in the category of (differential graded) Lie algebras (called in [14] semi-simplicial Lie algebras or selas for short). In [13] we developed the deformation theory of Lie atoms, and this was extended in [14] to the case of a sela. In any case, the deformation theory connection is based on interpreting bracketinduced maps on cohomology as obstructions. For this paper, an important example of a Lie atom is the shift $N[-1]$ where $N$ is the normal bundle of a submanifold $Y \subset X$ of a compact complex manifold, realized as the mapping cone of the inclusion

$$
i: T_{X / Y} \rightarrow T_{X}
$$

where $T_{X / Y}$ denotes the sheaf of holomorphic vector fields tangent to $Y$ along $Y$. Then the deformation theory of $N[-1]$ is the deformation theory of $Y$ as submanifold of $X$ (i.e. the local structure of the Hilbert scheme or Douady space), which goes back to Kodaira-Spencer and Grothendieck (see e.g. [15], §3.4.4). Its starting point is the classical obstruction map $\operatorname{Sym}^{2}\left(H^{0}(N)\right) \rightarrow H^{1}(N)$. An example of a sela is the sela $\mathcal{T}_{X}^{\cdot}$ associated to an algebraic scheme $X / \mathbb{C}$, whose deformation theory is that of $X$ as $\mathbb{C}$-scheme.

1.2. Jacobi-Bernoulli complex. Briefly put, associated to a sela $\mathfrak{g}$ satisfying suitable hypotheses (e.g. finiteness, vanishing of $\mathbb{H}^{0}$, where the latter corresponds to having trivial automorphisms), there is a comultiplicative complex $J^{\bullet}(\mathrm{g})$ in strictly negative degrees, called a Jacobi-Bernoulli complex, initially defined for Lie atoms in [13], $\S 1.2$, such that for the $(-n)$-th truncation $J_{n}(\mathrm{~g})$, we have that

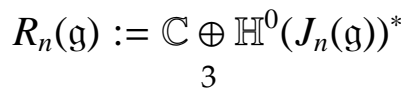


is a (commutative associative) Artinian local ring classifying $n$-th order deformations of $\mathfrak{g}$. Essentially, the (-i)-th term of $J$ is $\bigwedge^{i}(\mathfrak{g})$; however when $\mathfrak{g}$ is not a dgla (i.e. when the simplicial complex in question is not reduced to a point), some of the differentials in the complex must be twisted by Bernoulli numbers (hence the name). See e.g. [13], Thm. 3.3 (which is sufficient for this paper) and [14], Thm 1.3 and Thm. 6.1.

1.3. $T^{1}$ - lifting. Many results on unobstructed deformations, including those in this paper, rely on a connection with Hodge theory which has appeared before in a number of guises. Perhaps the most transparent one is the ' $T^{1}$-lifting criterion' of [12]. In a nutshell, this runs as follows. Suppose we have 'suitable' isomorphisms

$$
\phi_{i}: T^{i} \rightarrow H^{i}, i=1,2
$$

from a deformation group (resp. obstruction group) to a Hodge cohomology group. Let $v \in T^{1}$. Then the obstruction $o(v) \in T^{2}$ to lifting $v$ to higher order coincides via $\phi$ with the obstruction $\left\langle v, \phi_{1}(v)\right\rangle$ to deforming the cohomology class $\phi(v)$ in the direction $v$, i.e.

$$
\phi_{2}(o(v))=\left\langle v, \phi_{1}(v)\right\rangle
$$

(in the cases where this is used, both sides are computed in terms of Lie brackets, which ultimately turn out to be the same bracket). However, the RHS above vanishes as $\phi(v)$ is essentially topological in character (this requires the deformation corresponding to $v$ to be locally trivial, which holds in our case). Therefore $o(v)=0$. Thus, the deformation space corresponding to $T^{1}$ is unobstructed.

This heuristic reasoning is made precise in various references, including [12], [11], [16].

\section{THE NORMAL DG ATOM}

See [2] or [4] for Poisson basics.

2.1. Normal DG atom and Lagrangian deformations. A PL triple $(X, \Pi, Y)$ by definition consists of a a log-Lagrangian submanifold $Y$ of a pseudo-symplectic manifold $(X, \Pi)$. Fixing a PL triple $(X, \Pi, Y)$, our purpose in this section is to describe a Lie-theoretic object (a dg Lie atom) which controls log-Lagrange deformations of 
$Y$, i.e. deformations of $Y$ in $X$, fixing $X$, preserving the log-Lagrangian property. In the next subsection, this will be extended to deformations of the entire PL triple.

Let $N$ denote the normal bundle of $Y$ and let

$$
N^{\bullet}=\bigoplus_{i=1}^{n} \bigwedge^{i} N
$$

be the exterior algebra on $N$. As discussed above, it is classical (and independent of any Poisson or Lagrangian properties) that $N$ has a bracket structure that controls Hilbert deformations of $Y$ in $X$. From the Lie-atomic viewpoint, this is developed in detail in [13] (see especially §3.2, Example 1.1.4.D). In this section we will develop the log-Lagrangian analogue, where the main new feature is the differential on $N^{\bullet}$. Thus we will prove (compare [1]):

Theorem 1. Notations as above,

(i) $N^{\bullet}$ admits the structure of differential graded Lie atom;

(ii) if $Y$ is compact, $N^{\bullet}$-deformations coincide with Hilbert-Lagrange deformations of $Y$ in $X$, and the projection $N^{\bullet} \rightarrow N$ corresponds to the forgetful map from Hilbert-Lagrange to Hilbert deformations.

This result is not new. The existence of the differential on $N^{\bullet}$ was certainly known to Baranovsky et al. [1], as was, in some form, the relationship of $N^{\bullet}$ to log-Lagrangian deformations.

Proof of Theorem. (i) As mentioned above, the Lie atom structure of $N$ holds generally without any Poisson or Lagrange conditions, and was discussed in [13]. It is deduced from viewing it as the (shifted) mapping cone of the inclusion of Lie algebra sheaves

$$
T_{X / Y} \rightarrow T_{X}
$$

where $T_{X / Y}$ denotes the torsion-free sheaf of vector fields on $X$ tangent to $Y$. This structure induces a graded Lie atom structure on $N^{\bullet}$, deduced from the mapping cone of

$$
T_{X / Y} T_{X}^{\bullet} \rightarrow T_{X}^{\bullet}
$$

where $T_{X}^{\bullet}$ is the Schouten graded Lie algebra and $T_{X / Y} T_{X}^{\bullet}$ the exterior ideal generated by $T_{X / Y}$, which is easily seen to be a graded Lie subalgebra, though not a Lie ideal.

The Poisson structure $\Pi$ and log-Lagrangian condition on $Y$ enter into the differential (on $T_{X}^{*}$, hence on $N^{*}$ ). To see that the differential of $T_{X}^{*}$, i.e. [., $\Pi$ ], descends to $N^{\bullet}$ is suffices to show that the subalgebra $T_{X / Y} T_{X}^{\bullet}$ is closed under [., $\Pi$ ], and by elementary properties of the Schouten bracket it suffices to prove closedness of $T_{X / Y}$, 
i.e. to show that

$$
\left[T_{X / Y}, \Pi\right] \subset T_{X / Y} T_{X} .
$$

To show this note that the latter subsheaf of $\wedge^{2} T_{X}$ consists precisely of the local bivectors that pair to zero with $d f_{1} \wedge d f_{2}$ for all $f_{1}, f_{2} \in \mathcal{I}_{Y}$. Then, let $v$ be a local vector field on $X$ tangent to $Y$ (i.e. preserving the ideal sheaf $\mathcal{I}_{Y}$ ), and let $f_{1}, f_{2}$ be local functions in $\mathcal{I}_{Y}$. Then by a standard formula of Lichnerowicz, we have

$$
\left\langle d f_{1} \wedge d f_{2},[v, \Pi]\right\rangle= \pm v\left(\left\{f_{1}, f_{2}\right\}\right) \pm\left\langle\left(d v\left(f_{1}\right) \wedge d f_{2}-d v\left(f_{2}\right) \wedge d f_{1}\right), \Pi\right\rangle .
$$

This vanishes on $Y$ by the Lagrangian condition, which shows that $[v, \Pi] \in T_{X / Y} T_{X} \subset$ $\wedge^{2} T_{X}$.

Assertion (ii) follows from the stronger result, Theorem 2 below.

2.2. Deformations of PL triples. We will denote the differential graded Lie algebra $T_{X / Y} T_{X}^{\bullet}$ seen above by $T_{X}^{\bullet}\{Y\}$. Thus in degree $i, T_{X}^{i}\{Y\}$ is the subsheaf of $\wedge^{i} T_{X}$ locally generated by sections of the form

$$
u \wedge v_{1} \wedge \ldots \wedge v_{i-1}
$$

with $u$ a section of $T_{X / Y}$ and the $v_{j}$ sections of $T_{X}$. By a Poisson-Lagrange deformation of a PL triple $(X, \Pi, Y)$ as above we mean a triple $(\tilde{X}, \tilde{\Pi}, \tilde{Y})$ so that $(\tilde{X}, \tilde{\Pi})$ is a Poisson deformation of $(X, \Pi),(\tilde{X}, \tilde{Y})$ is a deformation of $(X, Y)$, and $\tilde{Y}$ is log-Lagrangian (isotropic) with respect to $\tilde{\Pi}$. Dropping the last condition leads to (plain) Poisson deformations of $(X, \Pi, Y)$.

Theorem 2. Assume $X$ is compact. Then the deformation theory of $T_{X}^{\bullet}\{Y\}$ coincides with the Poisson-Lagrange deformation theory of the triple $(X, \Pi, Y)$.

Proof. Given the theory of $\S 1$, what's being asserted is that given a local Artinian algebra $R$, Poisson-Lagrange deformations of $(X, \Pi, Y)$ are in bijective correspondence with comultiplicative elements of the Jacobi- Bernoulli cohomology group $\mathbb{H}^{0}\left(J\left(T_{X}^{*}\{Y\}, R\right)\right.$. In proving this assertion, we may assume the corresponding assertions for the differential graded Lie algebras $T_{X}^{\bullet}$ and $T_{X / Y}$ with $R$ coefficients, as well as for $T_{X}^{\bullet}\{Y\}$ with coefficients in $R_{1}, \operatorname{dim}_{\mathbb{C}}\left(R_{1}\right)<\operatorname{dim}_{\mathbb{C}}(R)$, to be true. The compactness assumption on $Y$ ensures that the groups in question are all finite-dimensional.

Thus let $R_{1}=R /(\eta)$ where $\eta$ is in the socle $\operatorname{Ann}_{R}\left(\mathfrak{m}_{R}\right)$, and suppose given a deformation diagram

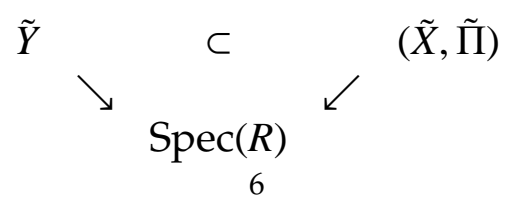


so that $(\tilde{X}, \tilde{\Pi})$ is a Poisson deformation, $(\tilde{Y} \subset \tilde{X})$ is a flat deformation, and so that the pullback over $R_{1}$ is a Poisson-Lagrange deformation. The obstruction to $\tilde{Y}$ being $\log$-Lagrangian over $R$ is the Poisson bracket

$$
\{., .\}: I_{\tilde{Y}} \times I_{\tilde{Y}} \rightarrow O_{\tilde{Y}}
$$

and by our assumption that the assertion above holds for $R_{1}$ in place of $R$, this map factors through a pairing

$$
I_{Y} \times I_{Y} \rightarrow O_{Y} .
$$

Note that the obstruction to $\tilde{Y}$ being log-Lagrangian is of a local nature, so in analyzing it we may choose compatible local coordinates on $X$ and $Y$ and assume that the deformations $\tilde{X}$ and $\tilde{Y}$ are, separately and not necessarily compatibly, trivial: i.e.

$$
\tilde{X} \simeq X \times \operatorname{Spec}(R), \tilde{Y} \simeq Y \times \operatorname{Spec}(R) .
$$

Then the pairwise deformation $\tilde{Y} \rightarrow \tilde{X}$ corresponds to a map

$$
\begin{array}{r}
v: \mathcal{I}_{Y} \rightarrow \mathrm{m}_{R} \otimes O_{Y}, v \in H^{0}(N) \otimes \mathrm{m}_{R} \\
\mathcal{I}_{\tilde{Y}}=\left\{f+v(f): f \in I_{Y} .\right\}
\end{array}
$$

Then in these terms the obstruction (2) is given by

$$
\left(f_{1}, f_{2}\right) \mapsto\left\{v\left(f_{1}\right), f_{2}\right\}-\left\{v\left(f_{2}\right), f_{1}\right\}-v\left(\left\{f_{1}, f_{2}\right\}\right) .
$$

(by our assumptions this is in $\eta O_{Y} \subset \mathrm{m}_{R} O_{Y}$ ). On the other hand, in terms of the Poisson differential [., $\Pi$ ], this is exactly $\left\langle[v, \Pi], d f_{1} \wedge d f_{2}\right\rangle$, QED.

\section{UNOBSTRUCTED DEFORMATIONS}

3.1. Set-up and statement. We will keep the notations of the previous section. Thus, $(X, \Pi)$ is a holomorphic Poisson manifold, not necessarily compact, and $Y$ is a compact log-Lagrangian submanifold. We now add the further hypotheses:

(i) $X$ is Kählerian and P-normal along $Y$, with Paffian divisor $D$;

(ii) $Y$ is transverse.

The transversality assumption means that $\bar{D}=D \cap Y$ has normal crossings. This is equivalent to the following condition: let $D^{[i]}$ denote the $i$-fold locus of $D$, which is locally a union of smooth branches of codimension $i$ in $X$. Then every branch of 
$D^{[i]}$ is transverse to $Y$, for all $i>0$. Note that this condition is strictly stronger than the condition that every local branch of $D$ itself is transverse to $Y$.

We recall that Poisson deformations (resp. Poisson-Lagrange deformations) of $(X, \Pi, Y)$ are deformations where $X$ deforms holomorphically, $\Pi$ deforms as Poisson structure, and $Y$ deforms as arbitrary (resp. log-Lagrangian) submanifold. The Poisson deformation space of $(X, \Pi, Y)$ coincides with the fibre product

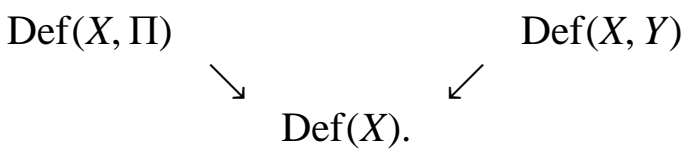

Here it is convenient to interpret these and similar deformation spaces as formal completions of suitable Kuranishi spaces. We denote by $\operatorname{Def}_{\text {loc. trival }}(X, D, Y)$ the space of deformations of the triple $(X, D, Y)$ where $D$ deforms locally trivially. This space corresponds to the dgla $T_{X}\{Y\}\langle\log D\rangle$.

Theorem 3. Notations as above, $Y$ has unobstructed Hilbert and Hilbert-Lagrange deformations in $X$, and these coincide; the space of first-order deformations of $Y$ in $X$ is canonically isomorphic to $H^{0}\left(Y, \Omega_{Y}^{1}\langle\log \bar{D}\rangle\right)$.

Furthermore, if $X$ is compact and P-normal, then the following assertions hold.

(i) The triple $(X, \Pi, Y)$ has unobstructed Poison-Lagrange and Poisson deformations and these deformations coincide and induce locally trivial deformations on $D$.

(ii) The deformation space $\operatorname{Def}_{\text {loc. trival }}(X, D, Y)$ is unobstructed.

(iii) There is a deformation space of quadruples $(X, \Pi, D, Y)$ that maps smoothly to $\operatorname{Def}(X, \Pi, Y)$ and to $\operatorname{Def}_{\text {loc. trival }}(X, D, Y)$

Remark 4. A map $D_{1} \rightarrow D_{2}$ of deformation spaces is smooth iff any infinitesimal deformation associated to $D_{2}$, parametrized by an Artinian (finite-dimensional) $\mathbb{C}$ algebra $R$, lifts to a deformation associated to $D_{1}$ and parametrized by $R$.

As in [11], we deduce directly from the Theorem:

Corollary 5. Assumptions as above with $X$ compact. Given a deformation $(\tilde{X}, \tilde{Y})$ of $(X, Y)$, the Poisson structure $\Pi$ extends to $(\tilde{X}, \tilde{Y})$ iff D extends locally trivially to $(\tilde{X}, \tilde{Y})$.

\subsection{Proof.}

Proof of Theorem. Let $\bar{D}$ be the restriction of the Pfaffian divisor $D$ on $Y$. By our hypotheses, both $D$ and $\bar{D}$ have normal crossings. Henceforth, we will denote by $\Omega^{\bullet}$ various de Rham complexes truncated to strictly positive degrees (i.e. omitting the zeroth term $\Omega^{0}=O$ ). Denote by $\Omega_{X}^{*}\{Y\}$ the kernel of the pullback map $\Omega_{X}^{\cdot} \rightarrow$ $\Omega_{Y}^{*}$. Thus, $\Omega_{X}^{1}\{Y\}$ is locally generated by $I_{Y} \Omega_{X}^{1}$ together with elements of the form 
$d f, f \in \mathcal{I}_{Y}$; and $\Omega_{X}^{\bullet}\{Y\}$ is generated by $\Omega_{X}^{1}\{Y\}$ as exterior ideal, i.e.

$$
\Omega_{X}^{i}\{Y\}=\Omega_{X}^{1}\{Y\} \wedge \Omega_{X}^{i-1}, i>1 .
$$

The basic formula

$$
[d f, d g]=d\{f, g\}
$$

shows that the log-Lagrangian hypothesis implies $\Omega_{X}^{1}\{Y\}$ is a Lie subalgebra of $\Omega_{X}^{1}$ under Poisson-Lie bracket. Then, using the usual derivation properties of brackets, it is not hard to check that $\Omega_{X}^{\bullet}\{Y\}$ has the structure of differential graded Lie algebra so that the inclusion into the Lie-Poisson algebra $\Omega_{X}^{*}$ is a Lie subalgebra. This turns the cokernel $\Omega_{Y}^{\bullet}$ into a differntial graded Lie atom. Likewise, for the log differentials $\Omega_{X}^{*}\{Y\}\langle\log D\rangle$, a subalgebra of $\Omega_{X}^{*}\langle\log D\rangle$ with cokernel atom $\Omega_{Y}^{*}\langle\log \bar{D}\rangle$. Now recall the homomorphism $\wedge^{\bullet} \Pi^{\#}$ already used in [11]. It yields a map of short exact sequences

$$
\begin{array}{ccccc}
0 \rightarrow & \Omega_{X}^{\bullet}\{Y\}\langle\log D\rangle \rightarrow & \Omega_{X}^{\bullet}\langle\log D\rangle \rightarrow & \Omega_{Y}^{\bullet}\langle\log \bar{D}\rangle \rightarrow & 0 \\
& \downarrow & \downarrow & \downarrow & \\
0 & T_{X}^{\bullet}\{Y\}\langle\log D\rangle \rightarrow & T_{X}^{\bullet}\langle\log D\rangle \rightarrow & N^{\bullet} \rightarrow & 0 .
\end{array}
$$

[Regarding the (possibly surprising) cokernel of the rightmost vertical map, note that the transversality of $Y$ and $D$ implies that the equations of the branches of $D$ at each point of $Y \cap D$ may be assumed to be part of a local coordinate system on $Y$, while the passage from $T_{X}^{\bullet}$ to $T_{X}^{\bullet}\{Y\}$ affects only coordinates normal to $Y$; hence the cokenel of $T_{X}^{\bullet}\{Y\}\langle\log D\rangle \rightarrow T_{X}^{\bullet}\langle\log D\rangle$ is the same as that of $T_{X}^{\bullet}\{Y\} \rightarrow T_{X^{\prime}}^{\bullet}$ i.e. $N^{\bullet}$. Heuristically, it is clear by transversality that motions of $Y$ in $X$ extend to (locally trivial) motions of $(Y, \bar{D})=(Y, Y \cap D)$ so the appropriate notion of 'lognormal complex' or " $N^{\bullet}\langle\log \bar{D}\rangle^{\prime \prime}$ is just $N^{\bullet}$.]

The first two vertical maps are dgla homomorphisms, hence the right vertical arrow is a Lie atom homomorphism. In any event, a local computation in [11] shows that the middle vertical arrow is bijective, and the same computation also shows that the left vertical arrow is bijective.

We will now prove (i). The argument is the same as in the proof of the main theorem in [11]: Delgne's $E_{1}$ degeneration theorem implies $E_{1}$-degeneration for $\Omega_{X}^{\bullet}\langle\log D\rangle$ and $\Omega_{Y}^{\bullet}\langle\log \bar{D}\rangle$, hence for $\Omega_{X}^{\bullet}\{Y\}\langle\log D\rangle$. Consequently, by a variant of the T1-lifting criterion reviewed in $\S 1$, the bracket pairing induces the trivial pairing on cohomology for the algebra $T_{X}^{\bullet}\{Y\}\langle\log D\rangle$, hence this algebra has unobstructed deformations. Indeed in this case the vanishing of obstructions is almost immediate from the fact that the exterior derivative operator $d$ induces the zero map on $\mathbb{H}^{\bullet}\left(\Omega_{X}^{\bullet}\{Y\}\langle\log D\rangle\right.$, plus the standard formula for Poisson-Lie bracket

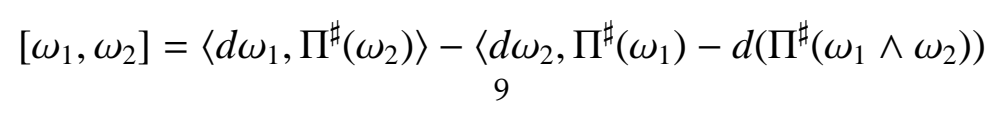


where $\Pi^{\sharp}$ denotes the duality operator (essentially interior multiplication by $\Pi$ ) which yields a null homotopy for the bracket-induced map

$$
\operatorname{Sym}^{2}\left(\Omega_{X}^{\bullet}\{Y\}\langle\log D\rangle\right) \rightarrow \Omega_{X}^{\bullet}\{Y\}\langle\log D\rangle .
$$

Then we see as in [11], §3.2, that the inclusion

$$
T_{X}^{*}\{Y\}\langle\log D\rangle \rightarrow T_{X}^{\bullet}\{Y\}
$$

is a direct summand projection, so that $(X, \Pi, Y)$ has unobstructed Poisson-Lagrange deformations. Specifically, we define a complex $K^{\bullet}\{Y\}$ by

$$
\begin{array}{r}
K^{0}\{Y\}=T_{X}\{Y\} ; \\
K^{1}\{Y\}=T_{X}^{2}\{Y\} \oplus N_{D}^{0} ; \\
K^{i}\{Y\}=T_{X}^{i+1}\{Y\} \oplus T_{X}^{i-1} \otimes N_{D}^{0}, i \geq 2
\end{array}
$$

where $N_{D}^{0} \subset N_{D}=O_{D}(D)$ is the image of $T_{X} \rightarrow N_{D}$, which also coincides with the image of $T_{X}\{Y\} \rightarrow N_{D}$ by transversality (NB: this explains why we don't need to define something like $N_{D}^{0}\{Y\}$ - it would be the same as $N_{D}^{0}$ ). The maps are as in [11], $\S 3.2$, as is the proof that the map $T^{\bullet}\{Y\}\langle\log D\rangle \rightarrow K^{\bullet}\{Y\}$ is a quasi-isomorphism. As there, we have a map $\phi: T^{\bullet}\{Y\} \rightarrow K^{\bullet}\{Y\}$ which yields a quasi-splitting to the inclusion $T^{\bullet}\{Y\}\langle\log D\rangle \rightarrow T^{\bullet}\{Y\}$. The components of $\phi$ going from $T_{X}^{i}\{Y\}$ to $T_{X}^{i}\{Y\}$ are the identity. The component of $\phi$ going from $T_{X}^{2}\{Y\}$ to $N_{D}^{0}$ is given by

$$
u \mapsto n u \wedge \Pi^{n-1} ;
$$

then as usual this is extended to $T_{X}^{i+1}\{Y\} \rightarrow T_{X}^{i-1} \otimes N_{D}^{0}, i>1$ as an exterior derivation, i.e. by

$$
v_{1} \wedge \ldots v_{i+1} \mapsto \sum_{a<b}(-1)^{a+b} v_{1} \wedge \ldots \wedge \hat{v_{a}} \wedge \ldots \wedge \hat{v_{b}} \wedge \ldots \wedge v_{i+1} \otimes \phi\left(v_{a} \wedge v_{b}\right) .
$$

The fact that Poisson-Lagrange and Poisson deformations coincide is a consequence of surjectivity of the edge map

$$
\mathbb{H}^{\bullet}\left(T_{X}^{\bullet}\{Y\}\langle\log D\rangle \rightarrow H^{\bullet}\left(T_{X / Y}\langle\log D\rangle\right)\right.
$$

which in turn is a special case of the $E_{1}$ degeneration. Indeed a Poisson deformation of $(X, \Pi, Y)$ induces a locally trivial deformation of $(X, D, Y)$, and the latter deformations are controlled exacly by the dgla $T_{X / Y}\langle\log D\rangle$. This proves assertion (i) in the Theorem.

As for assertions (ii) and (iii), consider the deformation space of quadruples $(X, \Pi, D, Y)$ that is associated to the dgla $T_{X}^{*}\{Y\}\langle\log D\rangle$. As we have seen, this is smooth as follows from $E_{1}$ degeneration for the latter complex. The fact that the inclusion $T_{X}^{\bullet}\{Y\}\langle\log D\rangle \rightarrow T_{X}^{\bullet}\{Y\}$ admits a left quasi-inverse, i.e. a left inverse in the 
derived category, implies its surjectivity in cohomology, whence smoothness of the induced map on deformation spaces. The smoothness of $\operatorname{Def}_{\text {loc.trivial }}(X, D, Y)$, and of the map to it from the deformation space of $T_{X}^{\bullet}\{Y\}\langle\log D\rangle$, follows from surjectivity on cohomology of the edge map

$$
\Omega_{X}^{\bullet}\{Y\}\langle\log D\rangle \rightarrow \Omega_{X}^{1}\{Y\}\langle\log D\rangle
$$

which is a consequence of Deligne's result on $E_{1}$ degeneration for $\Omega_{X}^{\bullet}\{Y\}\langle\log D\rangle$ [3]. This establishes assertion (ii) and (iii).

Finally, the smoothness and equality of Hilbert and Hilbert-Lagrange deformations of $Y$ assuming only compacness of $Y$ is a consequence of the diagram (3) (bijectivity of the right vetical arrow, which uses only P-normality of $X$ along $Y$ and transversality), plus Delgne's $E_{1}$ degeneration for $\Omega_{Y}^{*}\langle\log \bar{D}\rangle$, which implies the vanishing of obstructions for the dg Lie atoms $N^{\bullet} \simeq \Omega_{Y}^{*}\langle\log \bar{D}\rangle$ and $N \simeq \Omega_{Y}^{1}\langle\log \bar{D}\rangle$ and surjectivity of the edge map

$$
\mathbb{H}^{0}\left(N^{\bullet}\right) \rightarrow H^{0}(N) .
$$

\subsection{An example.}

Example 6. Let $S$ be a smooth compact surface and $C \subset S$ a smooth anticanonical divisor. Then $C$ corresponds to a Poisson structure $\Pi$ on S. As shown in [11], $\Pi$ induces a Poisson structure $\Pi^{[r]}$ on the degree-r Hilbert scheme $S^{[r]}$. This structure is not P-normal. Now let $r=2$. Then $\Pi^{[2]}$ still is not P-normal: its Paffian divisor is the locus of schemes having nonempty intersection with $C$, and has Whitney umbrella- type singularities; but $\Pi^{[2]}$ lifts to a P-normal Poisson structure $\Pi_{2}$ on the blowup $f: X_{2} \rightarrow S^{[2]}$ of $S^{[2]}$ in $C^{(2)}$, the locus of schemes contained in $C$. We saw in [11] that the deformation space of $\left(S^{[2]}, \Pi^{[2]}\right)$ is isomorphic to that of $\left(X_{2}, \Pi_{2}\right)$, hence is unobstructed. Briefly, the argument that the respective deformation spaces of $\left(S^{[2]}, \Pi^{[2]}\right)$ and $\left(X_{2}, \Pi_{2}\right)$ are isomorphic runs as follows. A deformation of $\left(S^{[2]}, \Pi^{[2]}\right)$ induces a locally trivial deformation of $\operatorname{Pf}\left(\Pi^{[2]}\right)$, hence a deformation of $C^{(2)}$ as the singular locus of $\operatorname{Pf}\left(\Pi^{[2]}\right)$, hence also a deformation of $\left(X, \Pi_{2}\right)$. Conversely, it is well known (e.g. [9] ) that a deformation of a blowup of a manifold along a smooth submanifold induces a a deformation of the blowdown morphism. In particular, a deformation of $X_{2}$ induces a deformation of $S^{[2]}$, therefore deformations of $\left(X, \Pi^{2}\right)$ induce deformations of $\left(S^{[2]}, \Pi^{[2]}\right)$.

Now let $B \subset S$ be a smooth curve transverse to $C$. Then $B^{(2)} \subset S$ is transverse to $C^{(2)}$, and $B^{2}=f^{-1}\left(B^{(2)}\right) \simeq B^{(2)}$ is transverse to $D=\operatorname{Pf}\left(\Pi^{2}\right)=f^{-1}\left(\operatorname{Pf}\left(\Pi^{[2]}\right)\right)$ and is log-Largrangian for $\Pi^{2}$. As noted above, the respective deformation spaces of 
$\left(S^{[2]}, \Pi^{[2]}\right)$ and $\left(X, \Pi^{2}\right)$ are naturally isomorphic, hence so are those of $\left(S^{[2]}, \Pi^{[2]}, B^{(2)}\right)$ and $\left(X_{2}, \Pi^{2}, B^{2}\right)$.

Therefore, $\left(S,{ }^{[2]}, \Pi^{[2]}, B^{(2)}\right)$ has unobstructed (Poisson, or equivalently PoissonLagrange) deformations.

For $r>2, \Pi^{[r]}$ is still P-normal along $B^{(r)}$, because locally a subscheme of $B$ can have at most a length-1 intersection with $C$; therefore by the Theorem, $B^{(r)}$ has unobstructed (Hilbert or Langrange: they are the same) deformations in $S^{[r]}$.

Conjecturally, as noted in [11], the rest of this example also extends to the case $r>2$, because it is conjectured that the following process yields a P-normal blowup of $\left(S^{[r]}, \Pi^{[r]}\right)$ : blowing up the $r$-fold locus of the Pfaffian of $\Pi^{[r]}$, then blowing up the proper transform of the $(r-1)$ st fold locus, etc. This conjecture is known to hold over the open set in $S^{[r]}$ consisting of curvilinear schemes (whose complement has codimension > 2). It appears to hold more generally over the set of locally monomial subschemes (defined locally by a monomial ideal); and this would seem to imply the general case since every subscheme is a deformation of a locally monomial one. The details of the case $r>2$ are yet to be written down.

Remark 7. Christian Lehn [10] has generalized the Voisin theorem to normal-crossing Lagrangian subvarieties $Y$. The analogous statement in the Poisson setting remains open (recall that in our treatment we are always assuming a Lagrangian $Y$ - unlike the Pfaffian divisor $D$ - to be smooth). 


\section{REFERENCES}

1. V. Baranovsky, Poisson deformation of coherent sheaves, talk at AMS special session on geometry of algebraic varieties, Riverside, Nov 2, 2013.

2. N. Ciccoli, From poisson to quantum geometry, Notes taken by P. Witkowski, avaliable on http://toknotes.mimuw.edu.pl/sem4/files/Ciccoli_fpqg.pdf.

3. P. Deligne, Théorie de Hodge III, Publ. Math. IHES 44 (1973), 5-77.

4. J.-P. Dufour and N. T. Zung, Poisson structures and their normal forms, Prog. Math., vol. 242, Birkhauser, Basel- Boston- Berlin, 2005.

5. R. Goto, Deformations of generalized complex and generalized Kähler structures, J. Differential Geometry $84(2010), 525-560$.

6. M. W. Gross, D. Huybrechts, and D. Joyce (eds.), Calabi-Yau manifolds and related geometries, Springer, 2003.

7. N. Hitchin, Deformations of holomorphic poisson manifolds, arxiv.org/1105.4775v1.

8. _ The moduli space of special Lagrangian submanifolds, Ann. Sc. Norm. Sup. Pisa Cl. Sci. 25 (1997), 503-515, revised version: arxiv.org: 9711002v1 [math.dg-ga].

9. E. Horikawa, Deformations of holmorphic maps III, Math. ann. 222 (1976), 275-282.

10. Christian Lehn, Deformations of lagrangian subvarieties of holomorphic symplectic manifolds, arxiv.org:1112.1887v2 [math.AG].

11. Z. Ran, Deformations of holomorphic pseudo-symplectic Poisson manifolds, arxiv.org/1308.2442.

12. , Deformation of manifolds with torsion or negative canonical bundle, J. Algebraic Geometry 1 (1992), 279-291.

13. L_L Lie atoms and their deformation theory, Geometric and Functional Analysis 18 (2008), 184221.

14. __ Jacobi-Bernoulli cohomology and deformations of schemes and maps, C. Europ. J. Math. 10 (2012), 1541-1591.

15. E. Sernesi, Deformations of algebraic schemes, Grundl. d. math. Wiss., vol. 334, Springer International, Berlin, Heidelberg, 2006.

16. C. Voisin, Sur la stabilité des sous-variétés lagrangiennes des variétés symplectiques holomorphes, Complex projective geometry (G. Ellingsrud, C. Peskine, G. Sacchiero, and S.A. Stromme, eds.), Cambridge university press, 1992, pp. 294-303.

UC MATH DEPT.

BIG SPRINGS ROAD SURGE FACILITY

RIVERSIDE CA 92521 US

ZIV.RAN @ UCR.EDU

HTTP : / /MATH.UCR.EDU/ ZIV/ 